\title{
Thermoacoustic Stability Boundaries in the Model Combustion Chamber with a Gas-Centered Swirl Coaxial Injector
}

\author{
Yong Ho Min, ${ }^{1}$ Chae Hoon Sohn $\mathbb{D}^{1}{ }^{1}$ and Youngbin Yoon ${ }^{2}$ \\ ${ }^{1}$ Department of Mechanical Engineering, Sejong University, Seoul 05006, Republic of Korea \\ ${ }^{2}$ School of Mechanical and Aerospace Engineering, Seoul National University, Seoul 08826, Republic of Korea \\ Correspondence should be addressed to Chae Hoon Sohn; chsohn@sejong.ac.kr
}

Received 31 March 2019; Revised 27 September 2019; Accepted 10 October 2019; Published 19 November 2019

Academic Editor: Joseph Morlier

Copyright (C) 2019 Yong Ho Min et al. This is an open access article distributed under the Creative Commons Attribution License, which permits unrestricted use, distribution, and reproduction in any medium, provided the original work is properly cited.

\begin{abstract}
The stability of a combustion chamber with a gas-centered swirl coaxial injector is investigated over a wide range of operating conditions in the aspect of thermoacoustic instabilities. First, flame shapes induced by the injector are analyzed for various recess lengths from experimental results. The spreading angle and flame size are reduced as the recess length increases. And, as a stability criterion, the damping factor is introduced. The operating conditions are divided into 7 sets with 9 tests, and fuel mass flow is fixed in each set. Stability boundaries are identified in terms of damping factor for the 63 tests by experiments without external perturbations. The stability map for the self-excited instability is obtained and it reveals two unstable regions. One is located at low dynamic head ratio and the other one is at high dynamic head ratio. A stable region is found at moderate dynamic head ratios. Relative stability is evaluated by various flow parameters over a wide range of operating conditions in the combustion chamber with a gas-centered swirl coaxial injector.
\end{abstract}

\section{Introduction}

The combustion chambers for rocket engines and aeroengines are designed with severe criteria. For example, the chamber should bear high mass flow, higher temperature, and higher combustion efficiency as well as more flexibility regarding operating conditions. However, these constraints have to be taken into account with the potential risk of thermoacoustic instabilities [1]. By nature, this nonlinear phenomenon appears between a stable and an unstable states [2]. The thermoacoustic instabilities can generate heat release rate fluctuation from flames, leading to large-amplitude pressure oscillations in a chamber. But, these oscillations cannot be withstood by the components of the chamber. Accordingly, combustion stabilization should be confirmed via postdesign, active control, and/or passive control to tackle this instability issue which can lead to serious damage to the chamber [3-5].

In most of cases, combustion stabilization is not attained over a full range of operating conditions, but limited to only a certain range of conditions, e.g., specific fuel and oxidizer mass flows [6,7], specific inlet fuel and oxidizer temperatures [8], specific chamber outlet boundary conditions [9], and specific fuels $[8,10-12]$. In practice, fuel and oxidizer mass flows are increased to produce high power output. The inlet fuel and oxidizer temperatures depend highly on the compressor upstream the combustion chamber. The inlet temperature can be also modified to adapt the overall energy output of the engine in which the combustion chamber is mounted. The combustion chamber outlet boundary condition is modified, for instance, if a turbine is placed downstream the combustion chamber. The fuels injected into the combustor chamber may vary according to users' needs. Currently, one demands a combustor to burn more flexible fuels such as syngas and oxy-fuel. All these factors influence combustion stability in a chamber. Therefore, in order to evaluate stability of a combustion chamber, stability criteria are investigated with respect to those factors. From the criteria, stability maps are identified and the stable operating ranges of the combustion chamber must be determined. The operating conditions of a chamber should be located in a stable regime where specific acoustic modes are not amplified, but suppressed. 


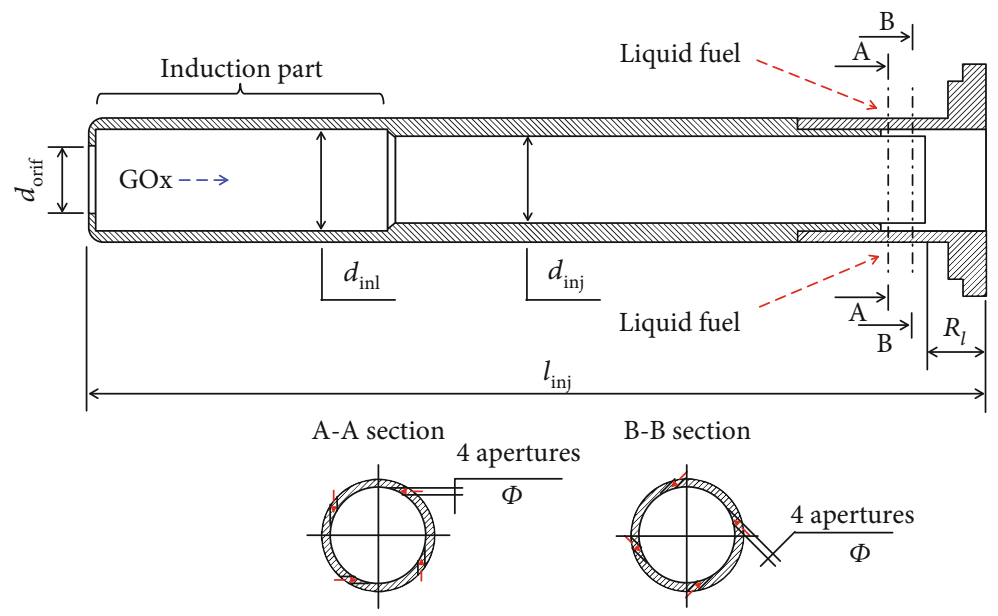

FIgURE 1: Diagrams of geometry of the model chamber and a single injector.

For understanding of instability phenomenon, many researchers have conducted numerical and experimental analyses $[13,14]$. Especially, detailed information on interaction between flows in the feeding line and in the combustor has been pursued. Depending on external source of perturbation, instabilities are divided into self-excited instability and forced instability. In the former, the instability occurs spontaneously without external perturbation $[15,16]$. On the other hand, forced instability is triggered by perturbation [7, 17]. A gas-centered swirl coaxial (GCSC) injector has been adopted widely in high-performance rocket engines, especially, with a staged combustion cycle. In this cycle, oxidizer-rich burning takes place in a preburner or a gas generator, and as a result, a small amount of product gas is produced. Much oxygen remains unreacted in a gas phase. Then, the mixture of unreacted gaseous $\mathrm{O}_{2}$ and the product gas flows into a turbine, which drives pumps. And then, it is injected into a chamber. Because of abundant $\mathrm{O}_{2}$, the mixture can be regarded as $\mathrm{O}_{2}$ approximately. A fuel is still liquid when it is injected. Although the GCSC injector is an important device for highperformance propulsion, there is little information on its stability characteristics.

In this regard, combustion stability is investigated over a wide range of fuel and oxidizer mass flows in a chamber with the GCSC injector in this study. The selected acoustic mode of interest is the second longitudinal mode in a chamber. Stability boundaries or unstable regions for self-excited instability are found in terms of the parameters of damping factor and then stability maps are demonstrated.

\section{Experimental Methods}

Experiments are conducted with a model chamber, not a full-scale chamber. To generate pure and clear longitudinal modes in a chamber, the model chamber has a cylindrical shape with the length, $L_{\mathrm{ch}, \mathrm{m}}$, of $537 \mathrm{~mm}$ and the diameter, $D_{\text {ch,m }}$, of $142 \mathrm{~mm}$. In this work with a model chamber, the second longitudinal (2L) mode is an interesting mode because the $2 \mathrm{~L}$ mode in a chamber with an open end corresponds to the $1 \mathrm{~L}$ mode in a chamber with both closed ends [18]. That is, the $2 \mathrm{~L}$ mode in the model chamber has the same waveform as the $1 \mathrm{~L}$ mode in the actual chamber. The length of the cylinder in a model chamber was determined by acoustic similarity rule for the second longitudinal mode [18] from the geometry of a baseline chamber [19]. Furthermore, the acoustic similarity for the longitudinal mode enables us to compute the length of the model chamber, $L_{\mathrm{ch}, \mathrm{m}}$, from the information on the equivalent cylindrical length of the actual chamber, $L_{\text {eff,a }}$, the speed of sounds in the model and the actual chambers, $C_{\mathrm{ch}, \mathrm{m}}$ and $C_{\mathrm{ch}, \mathrm{a}}$, respectively, and the Mach numbers, $M_{\mathrm{ch}, \mathrm{m}}$ and $M_{\mathrm{ch,a}}$ in the model and the actual chambers, respectively. The length of a cylindrical model chamber is calculated by the equation as follows:

$$
L_{\mathrm{ch}, \mathrm{m}}=1.5 L_{\mathrm{eff}, \mathrm{a}} \frac{1-M_{\mathrm{ch}, \mathrm{m}}^{2}}{1-M_{\mathrm{ch}, \mathrm{a}}^{2}} .
$$

In the model chamber, a single gas-centered swirl coaxial (GCSC) injector is mounted and has a fixed length, $L_{\text {inj }}$, of $117.2 \mathrm{~mm}$. The injector is divided into 2 parts, i.e., the gaseous oxidizer $\left(\mathrm{GO}_{\mathrm{x}}\right)$ passage in the center and the peripheral fuel inlet holes as shown in Figure 1. The detailed description of the injector can be found in the literatures $[19,20]$. In the present study, the oxidizer and the fuel mass flows are varied and thermoacoustic responses induced by such a change are investigated. As a geometric parameter of the injector, the recess length, $R_{l}$, is also a variable ranging from $4 \mathrm{~mm}$ to $12 \mathrm{~mm}$. The baseline length is $8 \mathrm{~mm}$.

Oxidizer and fuel densities and their velocities are specified at the inlets of the injector. The oxidizer density, $\rho_{\mathrm{o}}$, its velocity, $U_{\mathrm{o}}$, the fuel density, $\rho_{\mathrm{f}}$, and its velocity, $U_{\mathrm{f}}$, are adopted to define flow variables or parameters in this study. That is, two ratios are introduced in order to describe the variation of the oxidizer velocity with respect to the fuel velocity. The first ratio is the dynamic head ratio, $q$, which is defined as follows:

$$
q=\frac{U_{\mathrm{o}}}{U_{\mathrm{f}}} \sqrt{\frac{\rho_{\mathrm{o}}}{\rho_{\mathrm{f}}}} .
$$


TABLE 1: Flow conditions in all the test cases for experiments.

\begin{tabular}{lccccccc}
\hline Set no. & 1 & 2 & 3 & 4 & 5 & 6 & 7 \\
Test no. & $1 \sim 9$ & $10 \sim 18$ & $19 \sim 27$ & $28 \sim 36$ & $37 \sim 45$ & $46 \sim 54$ & $55 \sim 63$ \\
$U_{\mathrm{f}}(\mathrm{m} / \mathrm{s})$ & 5.86 & 7.81 & 9.76 & 11.74 & 14.3 & 15.62 \\
$U_{\mathrm{o}}(\mathrm{m} / \mathrm{s})$ & & & & $78.3 \sim 159.9$ & & & \\
$q$ & $6.18 \sim 12.62$ & $4.64 \sim 9.47$ & $3.71 \sim 7.57$ & $3.09 \sim 6.32$ & $2.53 \sim 5.17$ & $2.32 \sim 4.73$ & $2.06 \sim 4.21$ \\
MFR & $38.2 \sim 159.3$ & $21.5 \sim 89.7$ & $13.8 \sim 57.4$ & $9.6 \sim 39.9$ & $6.42 \sim 26.8$ & $5.4 \sim 22.42$ & $4.3 \sim 17.7$ \\
\hline
\end{tabular}

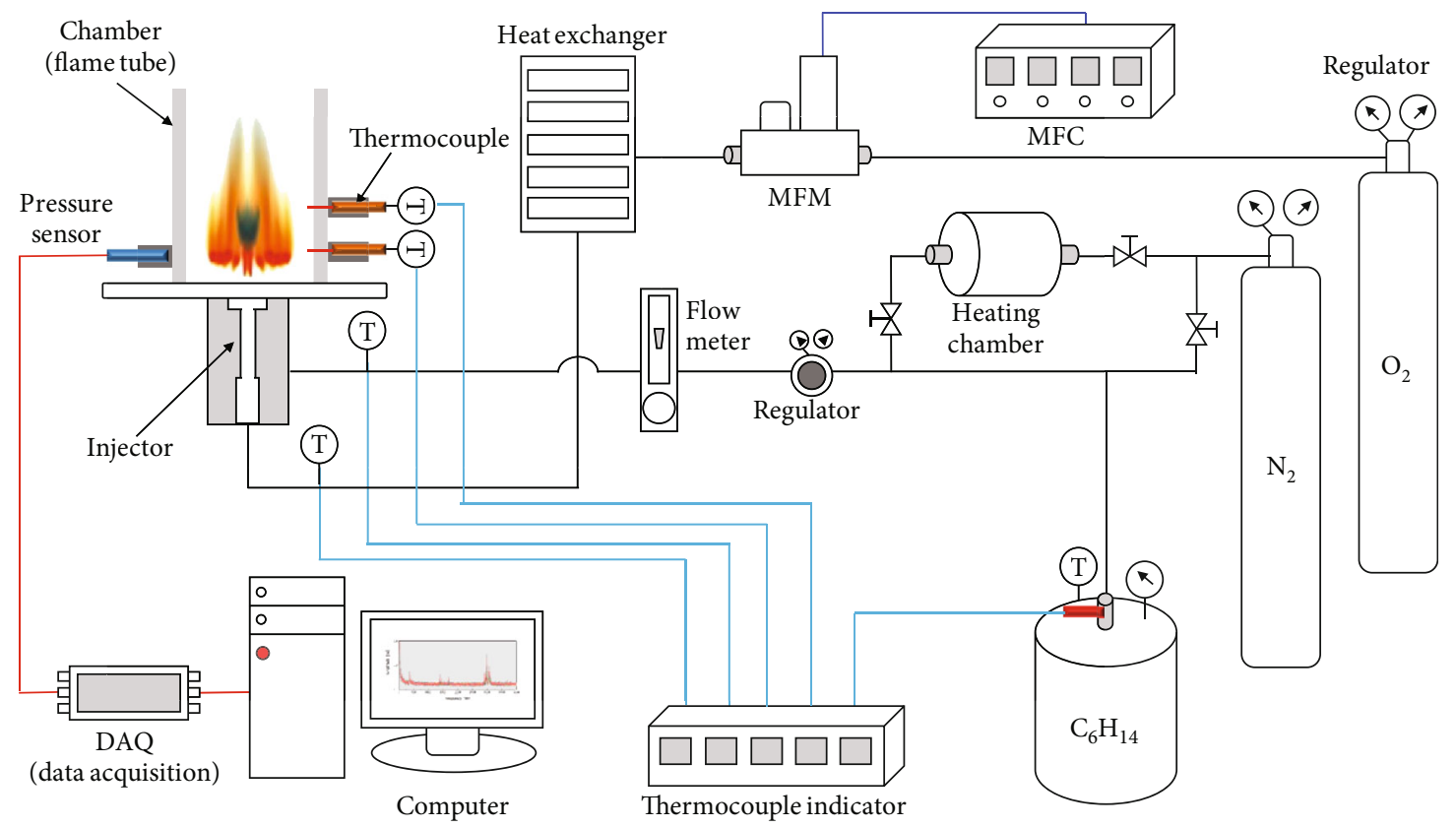

Figure 2: Schematic diagram of experimental apparatus.

From the definition, $q$ depicts the ratio of square roots of the kinetic energies at the inlets for the oxidizer and the fuel. Similarly, the momentum flux ratio, MFR, is also used to estimate the ratio of the oxidizer to the fuel momentum [20]. It is a more widely used flow parameter in the GCSC injector and is defined as follows:

$$
\operatorname{MFR}=\frac{\rho_{\mathrm{o}} U_{\mathrm{o}}^{2}}{\rho_{\mathrm{f}} U_{\mathrm{f}}^{2}} .
$$

From the definitions of the two ratios, they are the same as each other fundamentally.

The present study covers 63 test nos. or cases which are divided into 7 sets in experiments. In each set, the fuel velocity is fixed while the oxidizer velocity is changed with 9 increasing values. This means that the mixture gets leaner from the first test case to the last one in a set. Flow conditions in all the test cases are summarized in Table 1, and the design condition in the test is numbered 40 , that is, denoted by test no. 40 where flow rates are $U_{\mathrm{f}}=14.3 \mathrm{~m} / \mathrm{s}$ and $U_{\mathrm{o}}=109 \mathrm{~m} / \mathrm{s}$, respectively, from the previous work [21].
The schematic diagram of the experimental apparatus is shown in Figure 2. Vaporized n-hexane and oxygen are selected as simulants for fuel and oxidizer, respectively. Instead of kerosene fuel, n-hexane is adopted to facilitate experiments with liquid fuel because kerosene is a mixture, not a pure substance, and has higher vaporization temperature. Furthermore, the material properties of n-hexane are well known and close to those of kerosene [22]. And, the heating values of hexane and kerosene are similar to each other.

Flow rate of oxidizer is controlled by mass flow controller (MFC) and mass flow meter (MFM). Fuel, liquid hexane, is heated and vaporized in a cylindrical chamber, and the flow rate of vaporized hexane is controlled by a flow meter and a regulator. Nitrogen is also heated in a heating chamber to prevent fuel vapor from being condensed by nitrogen. For measurement devices, the experimental apparatus is equipped with thermocouples to measure the oxidizer and fuel temperatures and a dynamic pressure sensor to measure pressure fluctuation in the combustion chamber. The sensor signals are recorded by the DAQ board. The experimental procedure starts with purging the pipe of oxidizer and fuel by nitrogen and then creating a flame by using methane for 


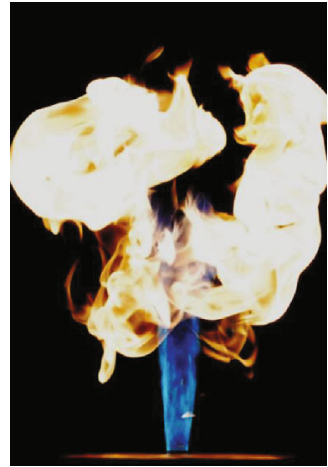

(a) Recess $4 \mathrm{~mm}$

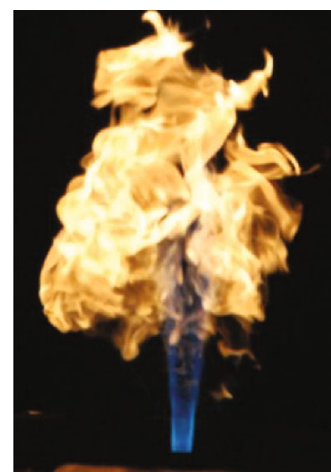

(b) Recess $8 \mathrm{~mm}$

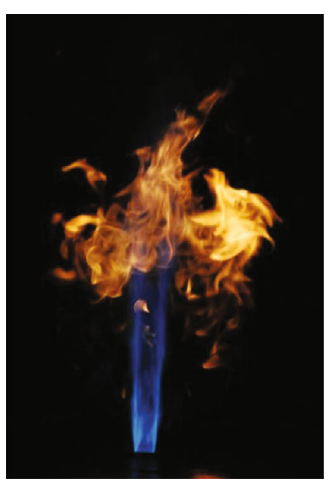

(c) Recess $12 \mathrm{~mm}$

Figure 3: Flame patterns for several recess lengths of (a) $4 \mathrm{~mm}$, (b) $8 \mathrm{~mm}$, and (c) $12 \mathrm{~mm}$ from the experiments of test no. 40 (MFR $=12.37$, equivalence ratio $=1.033, U_{\mathrm{f}}=14.3 \mathrm{~m} / \mathrm{s}$, and $U_{\mathrm{o}}=109 \mathrm{~m} / \mathrm{s}$ ).

ignition. Methane is supplied by an independent system of this apparatus although not shown in Figure 2. Fuel is then supplied through a flow meter, and the oxidizer is supplied in the next. Once the fuel and the oxidizer are properly supplied, methane is no longer supplied. When the specified quantities of the fuel and oxidizer are maintained, a DSLR camera takes pictures of flame. After a combustion chamber is put onto the injector face plate, dynamic behaviors of flame are investigated by measuring pressure fluctuation inside the chamber, which has an open end at the top. In order to measure the data, the 7 ports are made circumferentially around the chamber and dynamic pressure sensors and thermocouples are installed through the ports. The signal is recorded at the rate of 1,024 data per $0.125 \mathrm{~s}$. The FFT spectrum is calculated based on the signals. There is no imposed perturbation, and accordingly, self-excited instability is investigated here.

2.1. Damping Factor. The damping factor $[19,23]$ is a parameter which quantifies the acoustic-damping capacity in the system of an injector and a chamber. The factor, $\eta$, is defined as follows:

$$
\eta=\frac{f_{2}-f_{1}}{f_{\text {peak }}}
$$

where $f_{\text {peak }}$ is the frequency at which the peak response $\left(p_{\text {peak }}\right)$ appears and $f_{1}$ and $f_{2}$ are the frequencies at which the pressure amplitude corresponds to $p_{\text {peak }} / \sqrt{ } 2$ with $f_{2}>f_{1}$. This equation indicates that the damping factor becomes higher as the bandwidth is broadened on the FFT spectrum. Physically, the damping factor is proportional to the damping rate of pressure oscillation [23]. Pressure oscillation with a high damping factor means relatively stable oscillation. Accordingly, the factor is adopted as a parameter to quantify relative stability in this study.

2.2. Disturbance Wavelength. The disturbance wavelength, $\Lambda_{\mathrm{o}}$, is adopted to indicate the length of a disturbance wave propagating along the oxidizer jet at its velocity, $U_{\mathrm{o}}$ [24]. In determining $\Lambda_{\mathrm{o}}$, the frequency is chosen as that of the

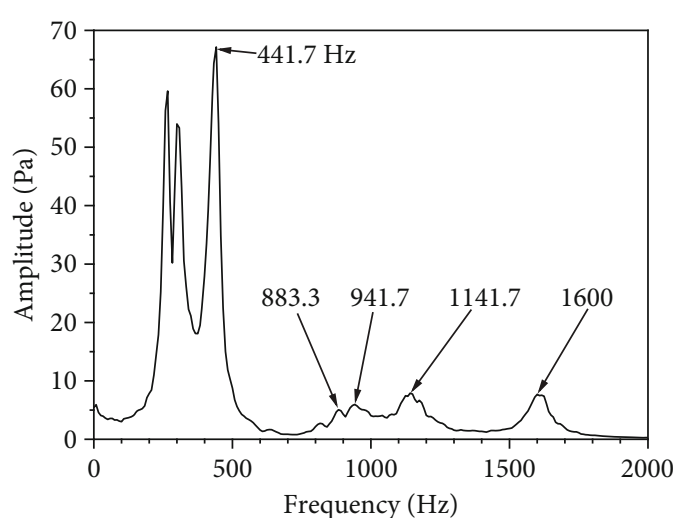

FIgURE 4: FFT spectrum for the design condition (test no. 40) with recess of $8 \mathrm{~mm}$.

2L-mode oscillation focused on this study. It is a parameter to compare the hydrodynamic wavelength with the characteristic lengths of jet and flame and defined as follows [24]:

$$
\Lambda_{\mathrm{o}}=\frac{u_{\mathrm{o}}}{f_{2 \mathrm{~L}}}
$$

In each case, oxidizer velocity is specified and the associated second longitudinal (2L) mode is generated at the frequency, $f_{2 \mathrm{~L}}$. The oxidizer velocity has a magnitude similar to the mean velocity in flow field in front of the flame front. Therefore, the disturbance wavelength has the meaning of the characteristic length of hydrodynamic disturbance coupled with the second longitudinal mode of the acoustic waves.

\section{Results and Discussion}

3.1. Flame Patterns. Firstly, flame patterns are examined for operating conditions as listed in Table 1. From the previous work [20], it has been found that the spray pattern varies depending on the recess length in numerical simulations. 


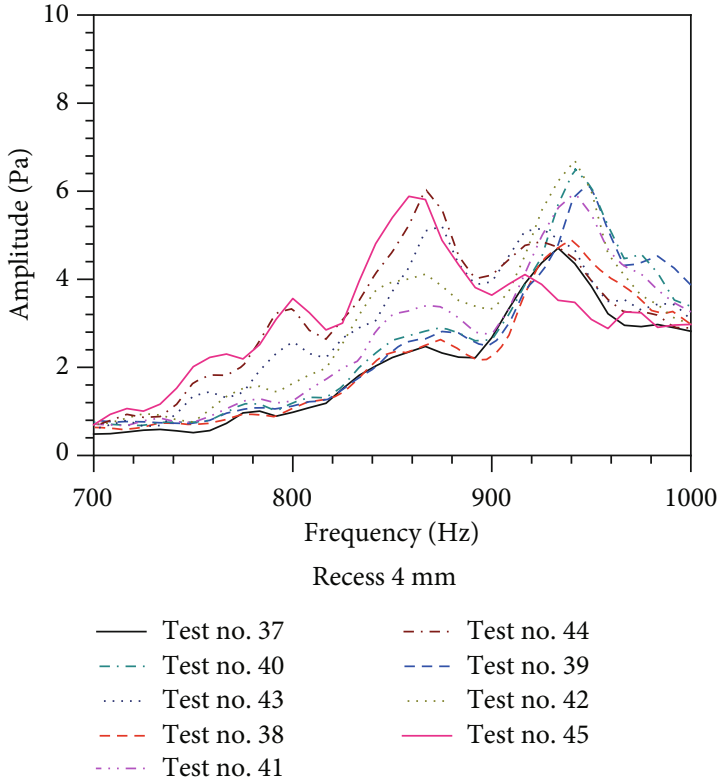

(a) recess $4 \mathrm{~mm}$

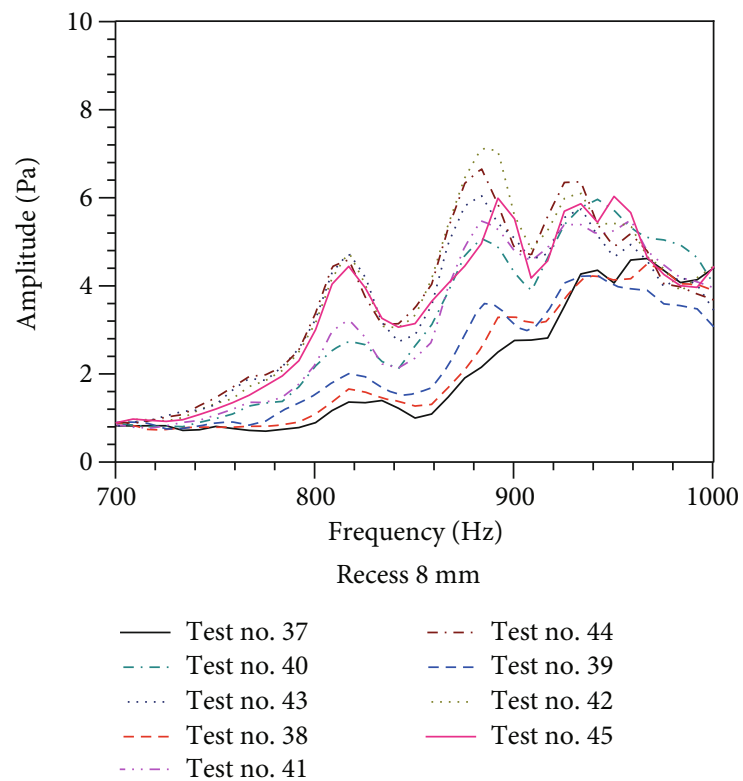

(b) recess $8 \mathrm{~mm}$

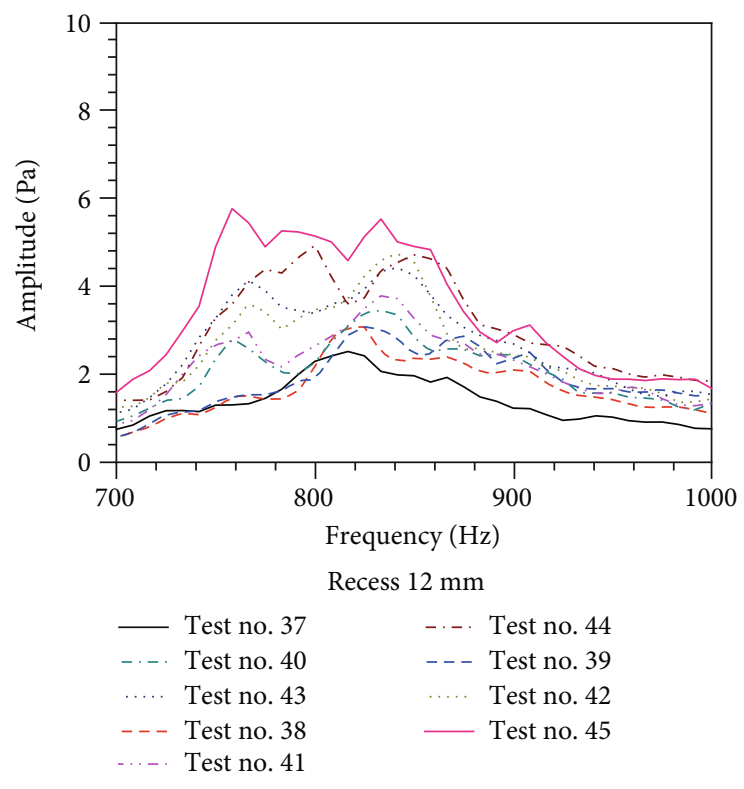

(c) recess $12 \mathrm{~mm}$

FIGURE 5: FFT spectrum in set no. 5 (test nos. 37-45) for various recess lengths.

As the recess length increases, the flame is stabilized and spreading angle of the jets decreases for the same condition. Accordingly, distribution of the propellants in the chamber is confined to a narrow zone and penetration length is elongated farther downstream. In this study, experiments are performed for the design operating condition of test no. 40 where MFR, oxidizer injection velocity, fuel injection velocity, and equivalence ratio are $12.37,14.3 \mathrm{~m} / \mathrm{s}, 109 \mathrm{~m} / \mathrm{s}$, and 1.033 , respectively.

For various recess lengths, $R_{l}$, from 4 to $12 \mathrm{~mm}$, flame patterns are shown in Figure 3, where direct photographs from experiments are demonstrated. The yellow flame size decreases with the recess length, which is induced by enhanced mixing of fuel and oxygen before the injector exit. With longer recess length, tangential velocity of the mixture stream in the injector decreases, resulting in reduced spreading angle of flame. But, change in the angle is not so significant. The angles at recess lengths of 4,8 , and $12 \mathrm{~mm}$ are $5.2,2.9$, and $2.0^{\circ}$, respectively. Although not shown here, at higher MFR, flame becomes narrow and longer downstream with a smaller spreading angle.

3.2. Effects of MFR and Recess Length on Pressure Oscillation. With the recess length of the injector fixed to be $8 \mathrm{~mm}$, pressure fluctuation signals are measured inside the chamber with fuel/oxidizer injection. From FFT spectrum in Figure 4, 
the frequencies of the acoustic resonant modes formed in the chamber are found for the design condition of test no. 40. The peak frequencies range from $441.7 \mathrm{~Hz}$ to $1600 \mathrm{~Hz}$. The two divided peaks observed at 266.7 and $300 \mathrm{~Hz}$ are not from acoustic resonance, but from the oxidant pipe connected vertically to the injector, which are not of interest here.

To identify acoustic modes of the five highest peaks from Figure 4, a theoretical equation for resonant frequencies is adopted. It is the correlation between frequency, sonic speed, and eigenvalue for each acoustic mode and it is expressed as follows [25]:

$$
f_{m, n, q}=\frac{c}{2}\left[\left(\frac{\alpha_{m n}}{R}\right)^{2}+\left(\frac{q}{L}\right)^{2}\right]^{1 / 2},
$$

where $c$ is the sound speed in the undisturbed gas, the subscripts $m, n$, and $q$ are termed wave numbers, and $\alpha$ and $q$ are eigenvalues. $R$ and $L$ are the radius and the length of the chamber, respectively. From Equation (6), the first peak with $441.7 \mathrm{~Hz}$ is identified as the first longitudinal (1L) mode and the second one with $883.3 \mathrm{~Hz}$ is the $2 \mathrm{~L}$ mode. As aforementioned, this study is focused on the $2 \mathrm{~L}$ mode, although it does not have the highest peak. Relative stability is evaluated here, which is little affected by absolute amplitude of a signal. The others with 941.7 and $1600 \mathrm{~Hz}$ are from the $1 \mathrm{~T}$ and the $2 \mathrm{~T}$ modes, respectively, and finally, the peak with $1141.7 \mathrm{~Hz}$ is predicted as the combined mode of $1 \mathrm{~T}$ and $2 \mathrm{~L}$. The sound speed, $c$, depends on temperature in a reactive field and the frequency is affected by the measured temperature in the experiment. Accordingly, theoretical frequency of the $2 \mathrm{~L}$ mode from Equation (6) should be corrected by applying the measured temperature.

In order to investigate effects of MFR and recess length on the 2L-mode fluctuation, the pressure amplitude of the 2L mode and its frequency are presented in Figure 5 for the set no. 5 with test nos. 37 to 45 including the design condition of test no. 40. And, recess length is changed from 4 to $12 \mathrm{~mm}$. As the MFR (or oxidizer velocity) increases from test no. 37 to test no. 45, the amplitude of the pressure oscillation increases generally and the frequency of the second longitudinal mode is almost constant irrespective of recess length. For example, with the recess length of $12 \mathrm{~mm}$ in Figure 5(c), the amplitude is increased from 2.5 to $6 \mathrm{~Pa}$ and the frequencies are found between 817 and $760 \mathrm{~Hz}$.

Therefore, increase in MFR (or oxidizer velocity) tends to increase the amplitude of pressure fluctuation and affects little the frequency. From the amplitudes in Figure 5, it seems that the recess length of $4 \mathrm{~mm}$ induces slightly more unstable burning than the other lengths of 8 and $12 \mathrm{~mm}$. To see the effects of the recess length on combustion stability quantitatively, damping factors are calculated for broad MFRs and shown in Figure 6. For the same flow conditions, the damping factor tends to increase with the recess length. With the shortest recess length of $4 \mathrm{~mm}$, the damping factors are the lowest over the full range of MFR, which means the most unstable burning. There is little difference between damping factors with the recess of 8 and $12 \mathrm{~mm}$. Accordingly, with the present injector adopted, shorter recess length than a critical

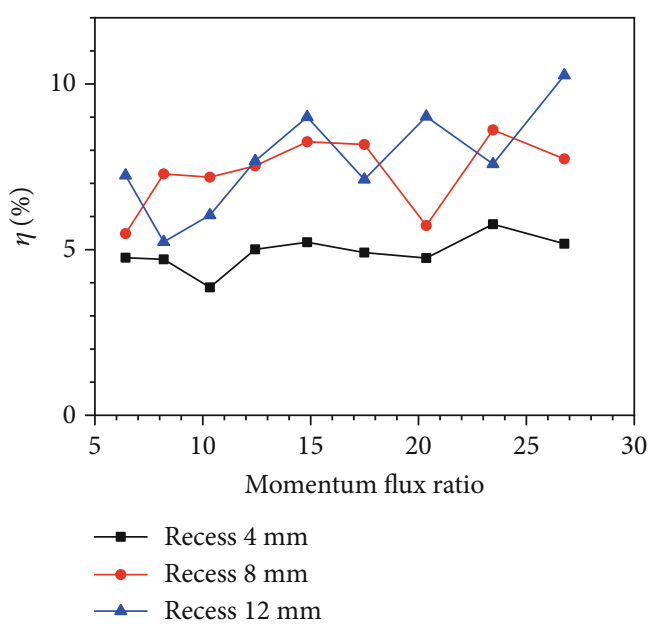

FIgURE 6: Damping factors in set no. 5 (test nos. 37-45) for various recess lengths.

one enhances combustion instability. And, the damping factor shows nonmonotonic behavior as a function of MFR. But, the effects of MFR on stability should be investigated comprehensively for all the operating conditions, which will be investigated in the later section.

3.3. Stability Maps for Self-Excited Instability. Acoustic resonance or damping for thermoacoustic instability is quantified by the damping factor defined in Equation (4) and then, from relative stability, stability map can be plotted, where unstable regions are demonstrated on the coordinate plane of flow parameters [26]. In the present study, the damping factors for all the 63 cases are evaluated. To plot stability map, the boundaries between stable and unstable regions should be found, which are called stability boundaries. They are found as follows. Damping factors for the set nos. 3 and 4 are evaluated and shown as a function of MFR, respectively, in Figure 7. In each set, the three unstable cases are circled by a dotted line, which means the test nos. have relatively low damping factors. This approach consists in selecting 3 test cases, where the damping factor is the lowest over the sets, in each set as a stability criterion. Then, the lines connecting the cases or test nos. with each other are considered as stability boundaries.

According to this criterion suggested here, stability boundaries between stable and unstable regions are found and are demonstrated on the coordinate plane of dynamic head ratio and disturbance wavelength in Figure 8, where two unstable regions are identified as a stability map. They are divided into unstable regions I and II. The unstable region I is located in the zone with high dynamic head ratios between 4 and 11. In this region, the disturbance wavelength is relatively long between $118 \mathrm{~mm}$ and $143 \mathrm{~mm}$, which means the hydrodynamic jets generated by the oxidizer stream have high speed. On the other hand, the unstable region II has relatively low dynamic head ratios between 2 and 6.5 and shorter disturbance wavelength between $65 \mathrm{~mm}$ and $114 \mathrm{~mm}$. Furthermore, this region includes the design condition. From this stability map, the stable region is located 


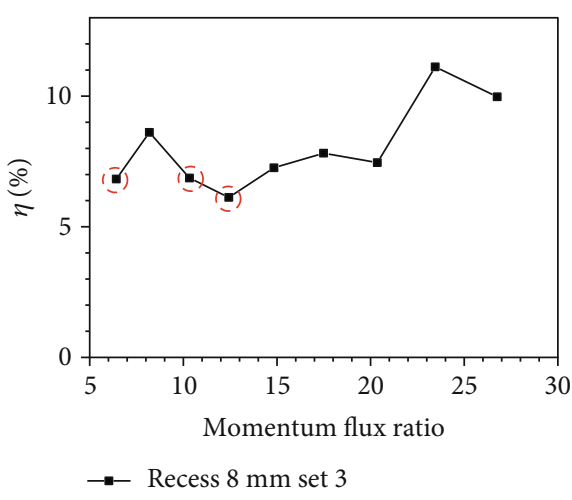

(a) Set no. 3

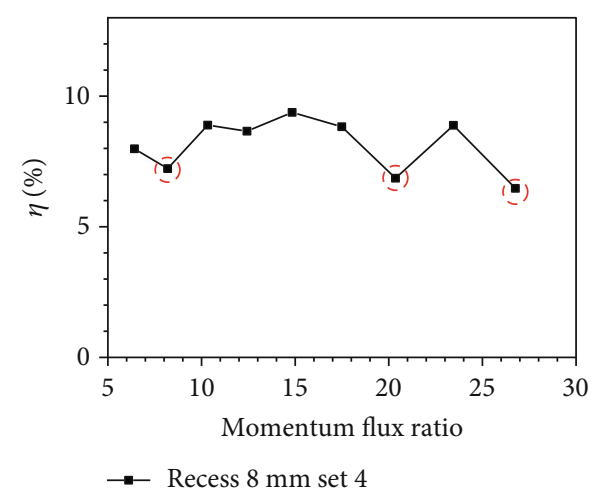

(b) Set no. 4

Figure 7: Damping factors for various test conditions in (a) set no. 3 and (b) set no. 4.

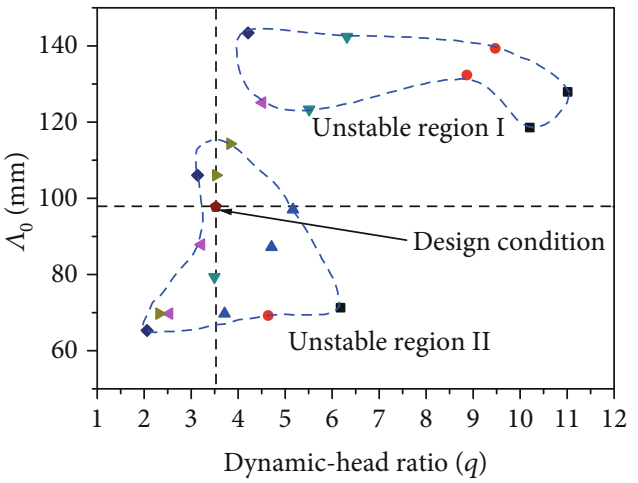

FIGURE 8: Instability region boundaries plotted on the coordinate planes of $q-\Lambda_{0}$.

between the two unstable regions with the ranges of dynamic head ratio from 6 to 10 and disturbance wavelength from $60 \mathrm{~mm}$ to $120 \mathrm{~mm}$. However, it is notable that this prediction is from relative stability only in a set. The stability map does not indicate that the operating conditions inside unstable regions result in combustion instability necessarily, but they are relatively more unstable than those outside the regions.

Stability map can be demonstrated more quantitatively by three-dimensional diagram including damping factor as seen in Figures 9 and 10, where comprehensive stability can be identified more clearly for all the conditions. Figures 9 and 10 show damping factors as functions of $q$ and $\Lambda_{\mathrm{o}}$ (or $U_{\mathrm{o}}$ ) and MFR and $\Lambda_{\mathrm{o}}$ (or $U_{\mathrm{o}}$ ), respectively. The two distinctive regions with relatively low damping factors, considered as unstable region, are identified, which are located at upper-right and lower-left zones, respectively. Their locations are close to those found in Figure 8, where stability boundaries are plotted according to the criterion aforementioned.

From Figure 9, unstable region I is identified in the region where both the dynamic head ratio and the disturbance wavelength have large values between 10 and 12 and between 120 and $140 \mathrm{~mm}$, respectively. The other region, region II, is relatively less appreciable and found in a narrow region where both the dynamic head ratio and the disturbance wavelength have small values between 2 and 3 and between $60 \mathrm{~mm}$ and $75 \mathrm{~mm}$, respectively. The stability map expressed by oxidizer inlet velocity, $U_{0}$, in a vertical axis has similar distribution of damping factor. Unstable oscillation in the region I would be induced by severely fluctuating flames under the preblowout condition with high jet speed [24]. But, the mechanism for the region II is different and the length of a disturbance wave propagating along the oxidizer jet at the jet velocity, $U_{0}$, should be linked with a characteristic length of burning zone, where heat release rate is the highest, although the characteristic length is not clarified in this study.

Sometimes, MFR is adopted as a flow parameter characterizing the GCSC injector instead of the dynamic head ratio, q. In Figure 10, damping factors are demonstrated as a function of MFR. As seen in Figure 9, there are two unstable regions and their locations are the same as in Figure 9. The unstable regions are found at small MFR between 4 and 10 and short disturbance wavelength between 65 and $78 \mathrm{~mm}$ as well as at large MFR between 80 and 120 and long disturbance wavelength between 120 and $140 \mathrm{~mm}$. The same conclusion can be drawn in terms of both dynamic head ratio and MFR as seen in Figures 9 and 10. On the other hand, the design condition is located away from the stability boundaries.

By this approach, i.e., evaluation from damping factor on the coordinate plane of two independent variables, comprehensive overview of stability can be seen rather than in the map demonstrated in Figure 8. The stability maps for selfexcited instability drawn by these two approaches reveal similar trends to each other, namely, two unstable regions located at low and high dynamic head ratios and a stable region located over a range of moderate dynamic head ratio between them.

\section{Conclusion}

The stability maps over a wide range of fuel and oxidizer mass flows have been experimentally pursued in the model chamber with a gas-centered swirl coaxial injector. This 


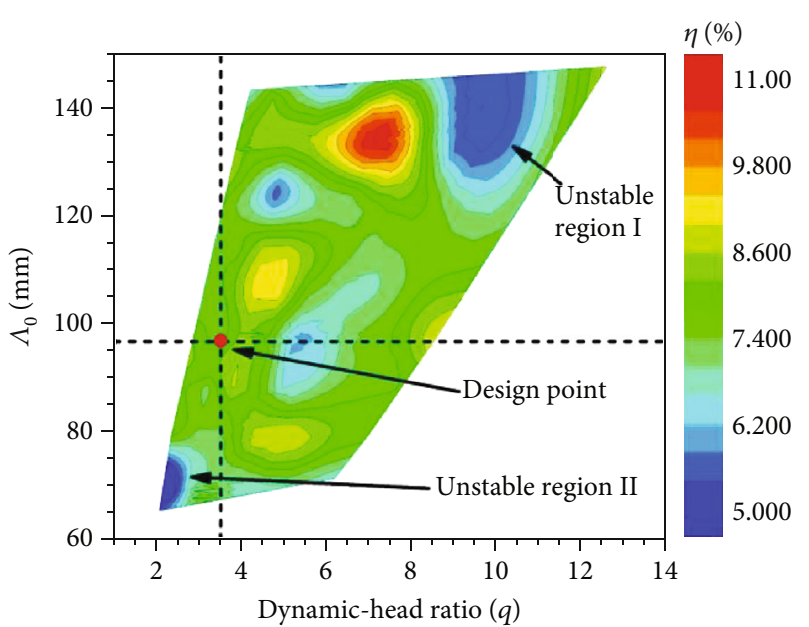

(a) $q-\Lambda_{\mathrm{o}}$

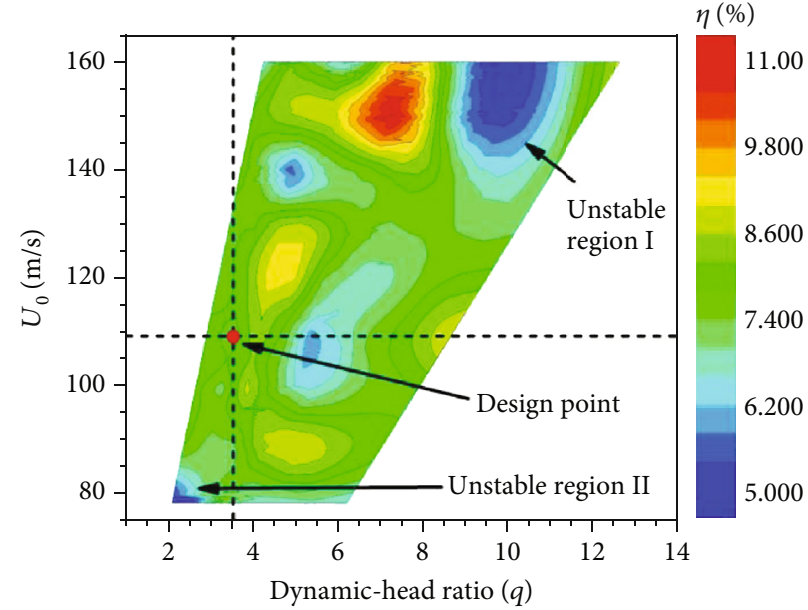

(b) $q-U_{\mathrm{o}}$

FIGURE 9: Damping factors plotted on coordinate planes of (a) $q-\Lambda_{\mathrm{o}}$ and (b) $q-U_{\mathrm{o}}$ under self-excited instability.

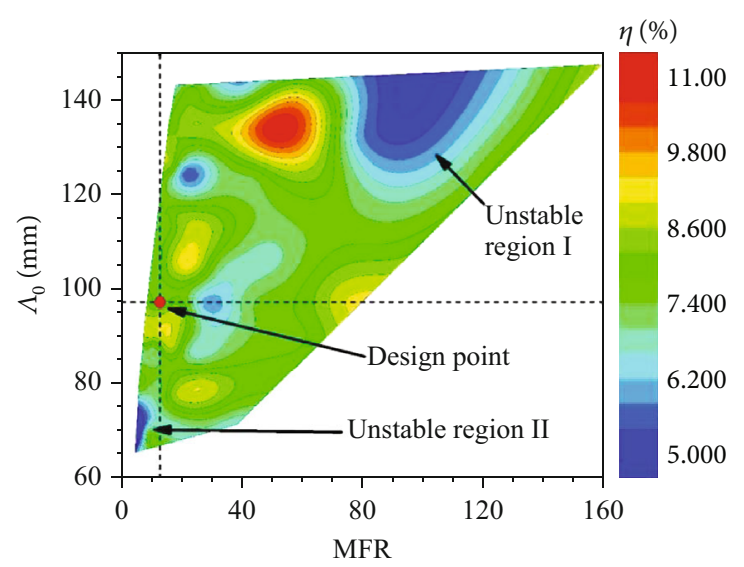

(a) MFR- $\Lambda_{\mathrm{o}}$

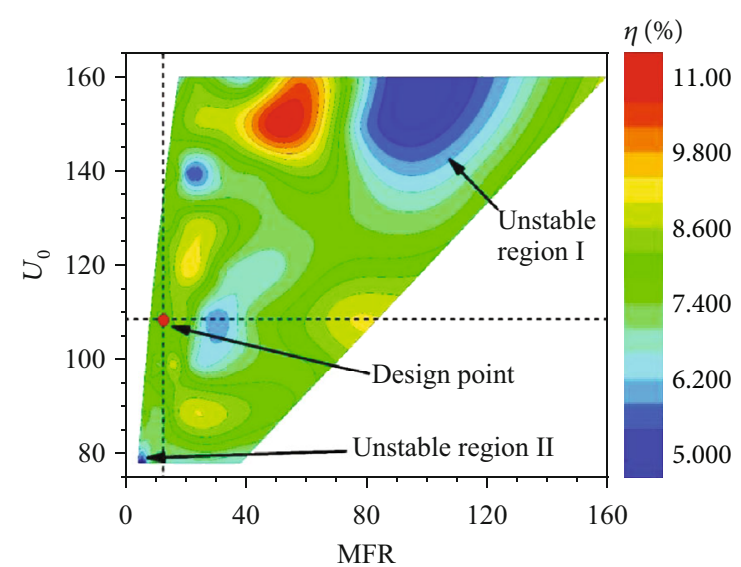

(b) MFR- $U_{\mathrm{o}}$

FIgURE 10: Damping factors plotted on coordinate planes of (a) MFR $-\Lambda_{\mathrm{o}}$ and (b) MFR $-U_{\mathrm{o}}$ under self-excited instability.

injector has been widely used in high-performance rocket engines with an oxidizer-rich staged combustion cycle.

First, flame patterns were compared with each other on the operating condition for various recess lengths. Damping factor has been selected as a stability criterion in the present study. The acoustic mode of interest is the second longitudinal mode. The study is focused on two aspects. One is to examine the effects of the oxidizer and fuel mass flows on generation of unstable combustion at the second longitudinal mode for various recess lengths. The other is to identify unstable regions on the stability maps with the recess length of $8 \mathrm{~mm}$. To determine stability boundary induced by selfexcited instability, the conditions with the 3 lowest damping factors are selected in each test set and they are demonstrated on the coordinate plane of dynamic head ratio and disturbance wavelength. In addition, three-dimensional diagram of damping factor is demonstrated for comprehensive overview of stability.

With respect to self-excited instability, there are identified two unstable regions located at low and high dynamic head ratios and a stable region located over the range of moderate dynamic head ratios. The most stable region is found over the ranges of a dynamic head ratio between 6 and 8 and a disturbance wavelength between $125 \mathrm{~mm}$ and $140 \mathrm{~mm}$ from three-dimensional diagrams. The possible instability mechanisms for two regions are from fluctuating flames and a characteristic length of burning zone with high heat release, respectively, but they are still to be pursued more clearly. The approaches proposed in this study can be a viable method to identify stability boundaries and applied to find stable and unstable regions, which provide the chamber designers with the data of the first approximation in 
determining a stable operating window at the initial stage of development of rocket combustors.

\section{Data Availability}

The numerical data in Figures 1 to 10 used to support the findings of this study are included within the article.

\section{Conflicts of Interest}

The authors declare that they have no conflicts of interest.

\section{Acknowledgments}

This work was supported by the Advanced Research Center Program (NRF-2013R1A5A1073861) through the National Research Foundation of Korea (NRF) grant funded by the Korean government (MSIP) contracted through Advanced Space Propulsion Research Center at Seoul National University.

\section{References}

[1] L. Rayleigh, “_The Explanation of Certain Acoustical Phenomena_ ", Nature, vol. 18, no. 455, pp. 319-321, 1878.

[2] K. T. Feldman Jr., "Review of the literature on Rijke thermoacoustic phenomena," Journal of Sound and Vibration, vol. 7, no. 1, pp. 83-89, 1968.

[3] X. C. Yuan and G. Keith, "Model refinement for the active control of thermo-acoustic instability," in 50th IEEE Conference on Decision and Control and European Control Conference (CDCECC), pp. 1686-1691, Orlando, USA, December 12-15, 2011.

[4] G. Daniel, R. Matthias, B. Mirko, and P. Christian, "Active control of combustion instability using pilot and premix fuel modulation," in International congress of Sound and Vibration (ICSV14), Cairns, Australia, July 9-12, 2007.

[5] C. Desjouy, G. Penelet, and P. Lotton, "Active control of thermoacoustic amplification in an annular engine," Journal of Applied Physics, vol. 108, no. 11, p. 114904, 2010.

[6] J. F. Van Kampen, R. A. Huls, J. B. W. Kok, and T. H. Van der Meer, "One dimensional acoustic modeling of thermoacoustic instabilities," Proceedings of the International Congress on Sound and Vibration, vol. 10, pp. 735-742, 2003.

[7] K. T. Kim and D. Santavicca, "Linear stability analysis of acoustically driven pressure oscillations in a lean premixed gas turbine combustor," Journal of Mechanical Science and Technology, vol. 23, no. 12, pp. 3436-3447, 2009.

[8] A. Patton, D. James, and I. Matthias, "Acoustic behavior of a partially-premixed gas turbine model combustor," in 50th AIAA Aerospace Sciences Meeting including the New Horizons Forum and Aerospace Exposition, Nashville, USA, January 0912, 2012.

[9] S. Roux, M. Cazalens, and T. Poinsot, "Outlet-boundarycondition influence for large eddy simulation of combustion instabilities in gas turbines," Journal of Propulsion and Power, vol. 24, no. 3, pp. 541-546, 2008.

[10] T. Lieuwen, V. McDonell, E. Petersen, and D. Santavicca, "Fuel flexibility influences on premixed Combustor Blowout, flashback, autoignition, and stability," Journal of Engineering for Gas Turbines and Power, vol. 130, no. 1, p. 011506, 2008.
[11] B. Chehroudi, "Recent experimental efforts on high-pressure supercritical injection for liquid rockets and their implications," International Journal of Aerospace Engineering, vol. 2012, 31 pages, 2012.

[12] C. T. Chang, K. Tacina, C. Lee et al., "NASA Glenn combustion research for aeronautical propulsion," Journal of Aerospace Engineering, vol. 26, no. 2, pp. 251-259, 2013.

[13] H. G. Sung and V. Yang, "Unified analysis of internal flowfield in an integrated rocket ramjet engine. II: ramjet sustainer," Journal of Aerospace Engineering, vol. 27, no. 2, pp. 398-403, 2012.

[14] Y. Zong, W. Bao, J. Chang et al., "Effect of fuel injection allocation on the combustion characteristics of a cavity-strut model scramjet," Journal of Aerospace Engineering, vol. 28, no. 1, p. 04014050, 2013.

[15] Y. Yu, J. C. Sisco, S. Rosen, A. Madhav, and W. E. Anderson, "Spontaneous longitudinal combustion instability in a continuously variable resonance combustor," Journal of Propulsion and Power, vol. 28, no. 5, pp. 876-887, 2012.

[16] A. P. Dowling, "Nonlinear self-excited oscillations of a ducted flame," Journal of Fluid Mechanics, vol. 346, pp. 271-290, 1997.

[17] J. Ranalli and D. Ferguson, "Measurement of flame frequency response functions under exhaust gas recirculation conditions," Journal of Engineering for Gas Turbines and Power, vol. 134, no. 9, p. 091502, 2012.

[18] C.H.Sohn, W.S.Seol, A. A. Shibanov, and V.P.Pikalov, "On the method for hot-fire modeling of high frequency combustion instability in liquid rocket engines," Journal of Mechanical Science and Technology, vol. 18, no. 6, pp. 1010-1018, 2004.

[19] C. H. Sohn, I. S. Park, S. K. Kim, and H. Jip Kim, “Acoustic tuning of gas-liquid scheme injectors for acoustic damping in a combustion chamber of a liquid rocket engine," Journal of Sound and Vibration, vol. 304, no. 3-5, pp. 793-810, 2007.

[20] Y. J. Kim, C. H. Sohn, M. Hong, and S. Y. Lee, “An analysis of fuel-oxidizer mixing and combustion induced by swirl coaxial jet injector with a model of gas-gas injection," Aerospace Science and Technology, vol. 37, pp. 37-47, 2014.

[21] C. H. Sohn, Y. J. Kim, Y. M. Kim, and V. P. Pikalov, “A scaling method for combustion stability rating of coaxial gas-liquid injectors in a subscale chamber," Journal of Mechanical Science and Technology, vol. 26, no. 11, pp. 3691-3699, 2012.

[22] S. P. M. Bane, J. L. Ziegler, P. A. Boettcher, S. A. Coronel, and J. E. Shepherd, "Experimental investigation of spark ignition energy in kerosene, hexane, and hydrogen," Journal of Loss Prevention in the Process Industries, vol. 26, no. 2, pp. 290 294, 2013.

[23] E. Laudien, R. Pongratz, R. Pierro, and D. Preclik, "Experimental procedures aiding the design of acoustic cavities," in Liquid Rocket Engine Combustion Instability, V. Yang and W. E. Anderson, Eds., vol. 169, pp. 377-399, AIAA, Washington, DC, 1995.

[24] C. H. Sohn, W. S. Seol, A. A. Shibanov, and V. P. Pikalov, "Combustion stability boundaries of the subscale rocket chamber with impinging jet injectors," Journal of Propulsion and Power, vol. 23, no. 1, pp. 131-139, 2007.

[25] M. J. Zucrow and J. D. Hoffman, Gas Dynamics, Wiley Inc, New York, 1976.

[26] R. Kaess, W. Polifke, T. Poinsot et al., "CFD-based mapping of the thermo-acoustic stability of a laminar premix burner," in Proceedings of the Summer Program, pp. 289-302, CA, USA, 2008. 


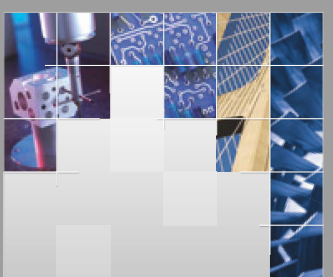

\section{Enfincering}
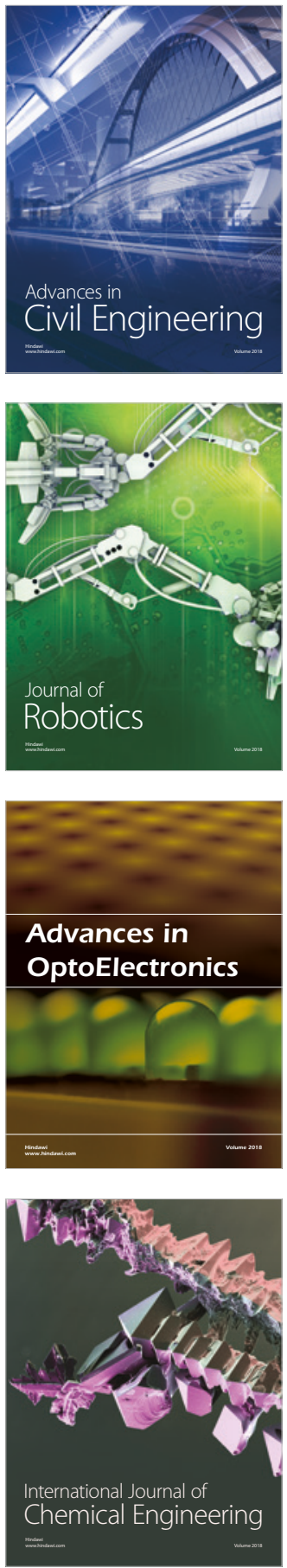

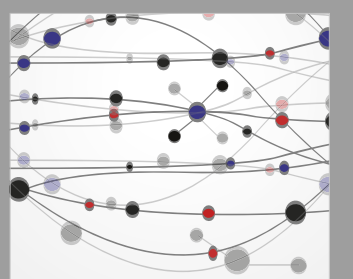

\section{Rotating \\ Machinery}

The Scientific World Journal

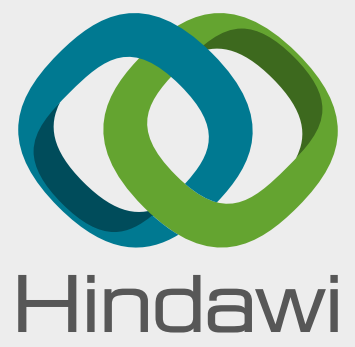

Submit your manuscripts at

www.hindawi.com
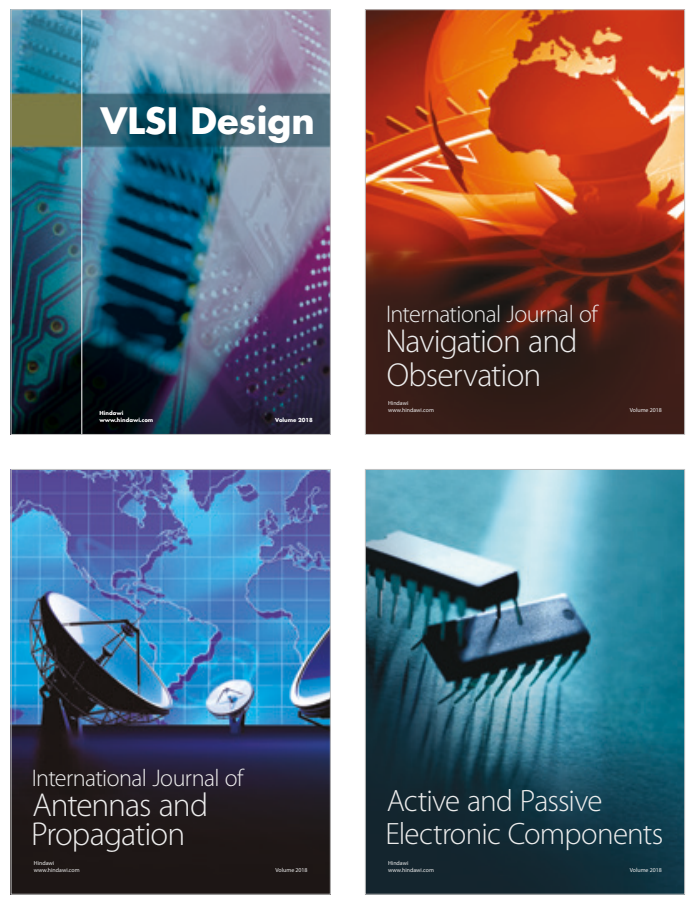
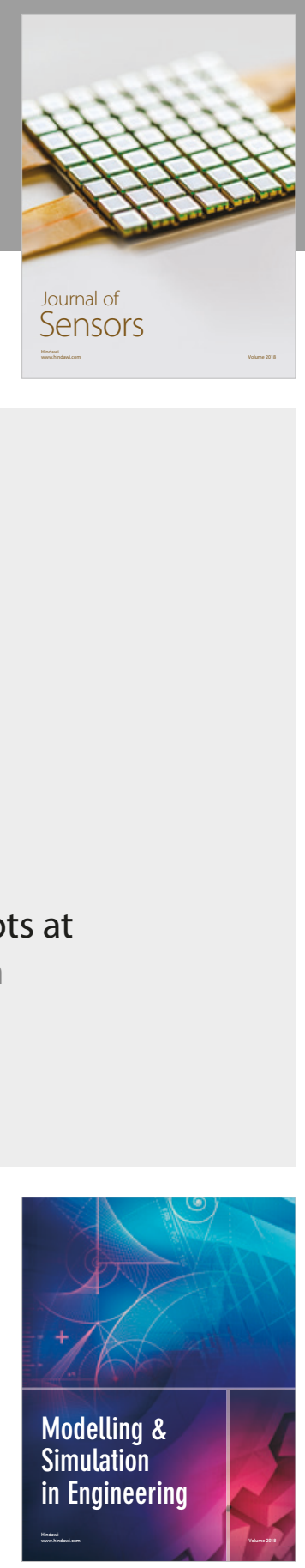

\section{Advances \\ Multimedia}
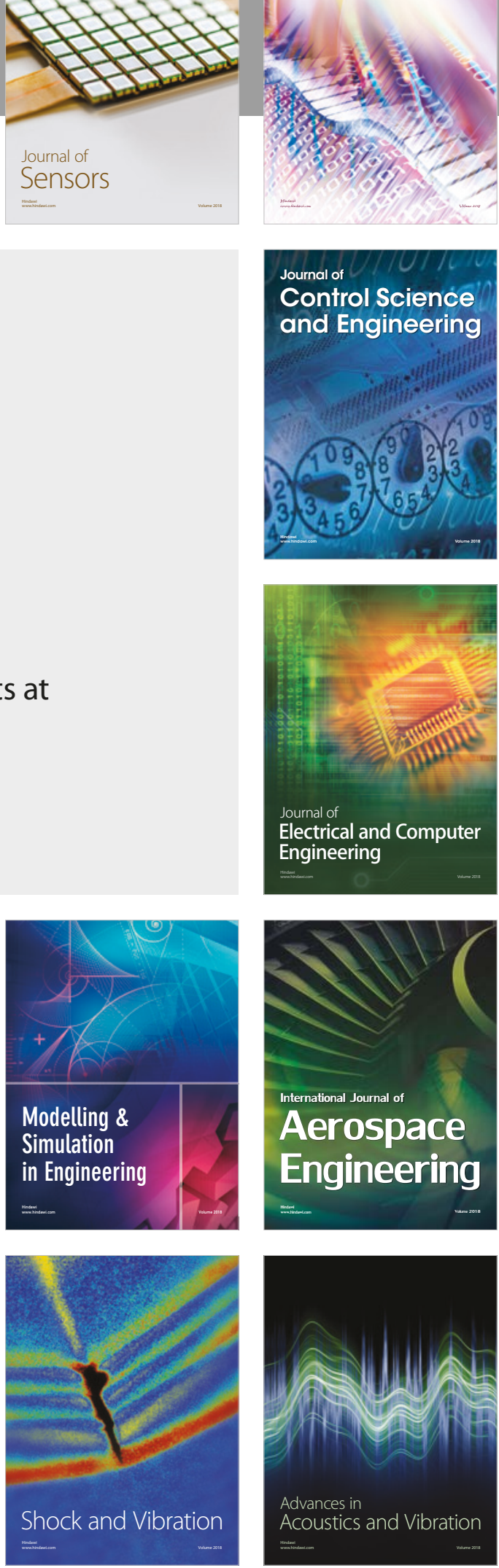